\title{
National immunisation days and vitamin A distribution in Mali: has the vitamin A status of pre-school children improved?
}

\author{
JF Schémann ${ }^{1, *}$, A Banou ${ }^{2}$, D Malvy ${ }^{3}$, A Guindo ${ }^{1}$, LTraore $^{1}$ and G Momo ${ }^{1}$ \\ ${ }^{1}$ Institute of African Tropical Ophthalmology (IOTA), BP 248 Bamako, Mali: ${ }^{2}$ Ophthalmic Centre, Segou, Mali: \\ ${ }^{3}$ Centre René Labusquière, University Victor Segalen Bordeaux 2, Bordeaux, France
}

Submitted 26 February 2002: Accepted 21 0ctober 2002

\begin{abstract}
Objectives: The impact on vitamin A status of the distribution of vitamin A during national immunisation days (NIDs) has not been well established despite strong promotion by international agencies and donors. Using a pre-post design, the change in prevalence of vitamin A deficiency was examined in pre-school children in Mali.

Design: Two cross-sectional surveys were conducted in Mopti region, the first in March 1997 before this strategy was adopted and the second in March 1999, four-anda-half months after a mass distribution of vitamin A during NIDs.

Subjects and setting: We compared the vitamin A status of children aged 12 to 66 months targeted in 1999 by NIDs with the status of children in the same age group in 1997. Infectious events of the previous two weeks were concurrently recorded. Within the 1999 sample, the status of recipient and non-recipient children was also compared.

Results: In 1997, the prevalence of xerophthalmia (defined by the presence of night blindness and/or Bitot spots) was 6.9\% (95\% confidence interval (CI) 5.1-9.2) and the modified retinol dose response (MRDR) test proved abnormal in $77.8 \%$ of $12-66-$ month-old children (95\% CI 68.27-85.17). In 1999 this picture had improved significantly, both for xerophthalmia prevalence, 3.3\% (95\% CI 2.1-5.2), and abnormal MRDR test response, 63.1\% (95\% CI 54.25-71.23). The infectious morbidity rates between 1997 and 1999 tended to decrease. No significant improvement was found among children older than those targeted by NIDs. In 1999, children who received vitamin A had a lower risk for xerophthalmia (3.0\% for recipients vs. $8.7 \%$ for non-recipients) and experienced fewer infectious events.

Conclusions: The clinical and biological vitamin A status of pre-school children improved between 1997 and 1999. Mass distribution of vitamin A appears to reduce the occurrence of xerophthalmia and would seem to be associated with a decrease in other related illnesses. Vitamin A supplementation during NIDs should be given a high priority when vitamin A deficiency remains a public health problem.

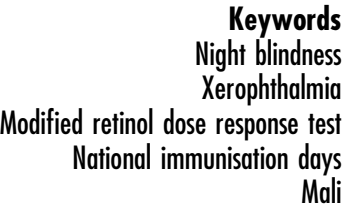

Keywords Night blindness Xerophthalmia National immunisation days

Mali
\end{abstract}

Vitamin A deficiency exists as a public health problem in 118 countries $^{1}$, with the highest prevalence of clinical deficiency occurring in Africa $^{2}$. Overall, biochemical deficiency is much more frequent and is responsible for increased mortality among children ${ }^{3}$. Since 1994, the World Health Organization (WHO) and the United Nations Children's Fund have recommended integrating the administration of vitamin A supplements with expanded immunisation programmes. In countries where routine immunisation is limited, the combination of vitamin A administration with polio vaccination campaigns has been adopted. National immunisation days (NIDs) target the population of children below 5 years of age and offer an optimal structure for delivering vitamin A supplements. In 1998, 40 countries had included vitamin A distribution during NIDs (22 African sub-Saharan countries) ${ }^{1}$. Twelve more countries joined the initiative in 1999. However, the impact of adding vitamin A distribution to NIDs has still not been well established.

Immunisation against paralytic poliomyelitis through NIDs is estimated to cost US\$ 1 per child and the average additional cost of vitamin $\mathrm{A}$ is evaluated at $5 \%$ of this amount. Indeed, the provision of high-dosage vitamin A supplements protects against the occurrence of xerophthalmia and blinding complications and also has an impact on children's general health, reducing the risk of mortality by $23 \% \%^{3,4}$. The reduction of child mortality attributable to vitamin A supplementation seems quite comparable to that of any vaccine administration $^{5}$ 
Mali is a sub-Saharan country where vitamin A deficiency remains a predominant public health condition $^{6}$. A survey carried out in 1997 in the Bandiagara Circle assessed the magnitude of the disorder. The findings were a strong argument for advocating vitamin A supplementation during Malian NIDs. The initiative was successfully applied across the entire country in December 1998 with a vitamin A coverage ratio close to 100\%. In order to examine the changes in vitamin A status in the child population less than 5 years old, a second survey was planned in March 1999 using a pre-post design.

\section{Methods}

The region of the Bandiagara Circle is situated in the centre of Mali. The population is involved mainly in agriculture and consists largely of Dogon, the largest ethnic group, living on the plateau and cliffs. Some groups of Peul, either nomadic or fairly sedentary cattle-breeders, can be found migrating during the dry season towards the Niger.

The study setting of the two surveys was similar. The two evaluations were planned in the Bandiagara Circle, in the same geographical, demographic and seasonal conditions during the same month of the year. In 1997 and 1999, both investigations were conducted in the same villages by the same team. The first survey was carried out in March 1997, before the strategy was adopted, and the second was conducted in March 1999, five months after NIDs. The NIDs allowed every child aged between 6 and 60 months to receive a vitamin A supplement (100000 IU for children under 12 months and 200000 IU for those over 12 months).

\section{Sampling metbods}

In the two original populations, different outcomes were documented to assess vitamin A deficiency: xerophthalmia defined by night blindness and/or Bitot spots, biochemical indicators of vitamin A status and stores, vitamin A intake, anthropometric features and illness symptoms.

The study sample was recruited using a cross-sectional, randomised, two-stage cluster sampling method, in accordance with the method advocated in the WHO blindness prevention programme ${ }^{7}$. The 1987 national census list of villages served as the sampling frame for the area $^{8}$. No urban or rural stratification was retained for the selection procedure. Twenty villages (clusters) were randomly selected. It was estimated that a sample of 1500 children aged between 6 months and 6 years was required, based on an expected prevalence of clinical deficiency (night blindness and/or Bitot spots) of 4\%, an alpha error of $5 \%$ and a desired absolute precision of $1 \%$. It was decided that 20 clusters of 75 children each would be selected, providing a total population of 1500 individuals. In each of the 20 villages a random plot was chosen and all of the neighbouring houses were visited gradually until the required number of children was reached. If a cluster was too small to recruit 75 children, the closest village was then chosen to complete the cluster.

Two sub-samples were drawn randomly from the original study population: one child out of every three for food intake inquiries and health status assessment (planned $n=500$ ), and from this latter group one out of every two individuals was selected randomly for biochemical status assessment (planned $n=250$ ).

\section{Opbthalmologic examination}

A history of night blindness (XN stage of xerophthalmia) was established using a questionnaire administered to the children's mothers. Three questions were sequentially asked in order to ascertain if her child was able to see efficiently in daytime, during the night and finally if night blindness was present (the vernacular term for the latter condition was used: in Dogon, gire nama and in the Peul language, pinku).

All children were given an ophthalmologic examination by the same ophthalmologist using $2.5 \times$ magnification and a lamp. Bitot spots (X1B) and active or non-active corneal lesions were noted. A categorical variable for xerophthalmia was derived corresponding to the presence of night blindness and/or Bitot spots.

At the end of the examination, vitamin A treatment (one capsule of 200000 IU, three times) was given to children showing clinical signs of vitamin A deficiency. Tetracycline ointment was applied to children with signs of ocular infection.

\section{Antbropometry}

Stature $(\mathrm{cm})$ and body weight $(\mathrm{kg})$ were measured by a trained nurse. Infants under 2 years of age were measured lying down and without shoes, using a conventional wooden height board. Individuals over 2 years old were measured standing up without shoes. Infants under 2 years of age were weighed using $25 \mathrm{~kg}$ Salter hanging scales (CMS Weighing Equipment, London, UK). Children over 2 years were weighed on scales calibrated before each session. Body weight was measured on children fully dressed (with light clothing) but without shoes.

Whenever possible, the child's age was verified on presentation of a birth certificate. Otherwise, it was estimated using a local calendar and history of past events.

These measurements allowed the calculation of normal standard deviation $(Z)$ scores. This was done for three anthropometric indices, based on reference values from the National Center for Health Statistics published by the $\mathrm{WHO}^{9}$ :

- weight-for-age (WAZ), reflecting weight insufficiency (underweight);

- height-for-age (HAZ), reflecting stunted growth or chronic malnutrition; and 
- weight-for-height (WHZ), reflecting wasting malnutrition.

$Z$-scores below the threshold of -2 were considered as moderate nutritional deficiency and less than -3 as severe nutritional deficiency.

\section{Assessment of foods rich in vitamin $A$}

A 7-day food-frequency questionnaire was administered to the mothers during the survey to estimate the number of occasions on which food sources of vitamin A and carotenoid were consumed. A list of 21 foods, six of animal origin rich in retinol and 15 of vegetable origin rich in $\beta$-carotene, that were available in markets, was compiled by trained local health practitioners. Sauces containing vegetables rich in $\beta$-carotene, such as baobab or gombo leaves, which are ingested in very small amounts, were given a weight considered as one-fifth of a serving in the intake analysis.

Specific vitamin A scores were calculated using the Helen Keller International (HKI) method. The 'animal score' was defined by adding the number of eating occasions per week based on animal vitamin A sources and the 'vegetable score' by summing the eating occasions per week based on vegetable sources. A community is considered to be at risk of vitamin A deficiency when the average value of the animal score is less than $4^{10}$.

\section{Morbidity events}

A two-week history of morbidity symptoms was recorded: episodes of coughing, fever or diarrhoea. Occurrence of measles was noticed if happening during the previous six weeks. Measles immunisation and the use of vitamin A capsules for the preceding three months were noted.

In 1999, mothers were asked if the examined child received vitamin A dosing during NIDs.

\section{Biochemical markers}

Vitamin A status was assessed by measuring serum retinol concentration and by use of the modified relative dose response (MRDR) test, an individual indicator of liver retinal stores ${ }^{11,12}$.

The detailed procedure for sample management has been described elsewhere ${ }^{11}$. Briefly, $200 \mu \mathrm{g}$ of acetate of 3,4-dehydroretinol (DR) was administered orally. Five hours later, $0.5 \mathrm{ml}$ of capillary blood was extracted by pricking the child's finger with a vaccination scratch blade and collected in a 'microtainer' tube (Beckton Dickinson). The tubes were set in a vacuum flask filled with ice and kept dark throughout the day. Within $3 \mathrm{~h}$, the tubes were centrifuged at room temperature ( $1300 \boldsymbol{g}$ for $10 \mathrm{~min}$ ). The serum was fractionated off, aliquots placed on ice for no more than $5 \mathrm{~h}$, frozen to $-20^{\circ} \mathrm{C}$ in a deep freeze at the nearest health centre before being taken to Bamako and then shipped on dry ice to Grenoble, France for laboratory analysis. The samples were analysed in April 1997 for the first survey and in April 1999 for the second.

Freezer tubes were thawed slowly to room temperature just before assay and $0.25 \mathrm{ml}$ aliquots of serum were extracted. Proteins were precipitated with absolute ethanol, and the fat-soluble vitamin A was subsequently extracted into a hexane phase. An automated highperformance liquid chromatography assay, made up of an isocratic system using silica gel (adsorption) as the stationary phase (Lichrosorb Si 60.5 column), was used for determination of DR and retinol. The retinol peak was detected spectrophotometrically at $313 \mathrm{~nm}$. The peaks were integrated by means of a Perkin-Elmer Sigma 10B Chromatography Data Station system.

The ratio of DR to retinol in the blood was calculated. The performance and reproducibility of this ratio has been evaluated in different population groups with vitamin A status classified as normal and abnormal ${ }^{12}$. Deficiency is defined as a ratio greater than or equal to 0.06 . A prevalence of abnormal tests greater than $20 \%$ defines the occurrence of a public health deficiency problem. The situation is considered to be severe when the prevalence is above $30 \%{ }^{13}$.

Control samples and external reference standards chosen in relation to storage duration and transport were used to assess the reliability of the analysis procedure. Cumulative quality control data for each analyte were provided during the analysis period. Briefly, the coefficients of variation for the estimation of retinol and DR were $3.3 \%$ and $3.0 \%$, respectively.

The thresholds used for serum retinol values in assessing vitamin A deficiency were $0.7 \mu \mathrm{mol} \mathrm{l}^{-1}$ and $0.35 \mu \mathrm{moll}^{-1}$, reflecting low status and severe biological deficiency, respectively ${ }^{13}$.

\section{Data management}

Data analysis was conducted as follows.

- The criterion of age recruitment for the two survey samples was for children aged 6 to 84 months in both 1997 and 1999.

- NID intervention was conducted in November 1998, five months prior to the evaluation, and restricted to children aged 6 to 60 months.

- Subsequently, the targeted sample of recipients was aged 12 to 66 months at the time of the 1999 survey. Thus it was necessary to stratify the two populations according to these age groups in order to evaluate the impact of adding vitamin A dosing during NIDs to the same age group of individuals.

- To this end, and to achieve an adequate comparison, the distribution of clinical and biochemical endpoints was estimated separately for children aged between 12 and 66 months and for individuals aged between 66 and 84 months, this latter age group not being covered by the programme. 
- Moreover, children below 24 months of age were not included in the analysis of night blindness or in the food survey, as these factors were not easy to determine and could not be declared accurately.

Data were recorded on standardised forms, reviewed daily for accuracy and completeness, and entered into Epi Info version 6.03 (Centers for Disease Control and Prevention, Atlanta, GA, USA). Categorical outcomes, such as prevalence rates, were initially compared by the chi-square test. Continuous data across groups were compared by analysis of variance if normally distributed. The Wilcoxon rank sum test was used for non-normally distributed data. Odds ratios (95\% confidence interval (CI) and related $P$-value) were calculated to compare status between groups.

\section{Etbical clearance}

The protocol was approved by the Institute of African Tropical Ophthalmology's Ethical Committee. A consent form written in French was translated for the mothers of the infants into either Dogon or Peul language and was signed by them prior to the examination.

\section{Results}

One thousand five hundred and ten children were included in the sample in 1997 and 1524 in 1999. In 1999, NIDs coverage of the targeted population was excellent since $94.5 \%$ of children aged from 12 to 66
Table 1 Populations in the two surveys

\begin{tabular}{lcc}
\hline & 1997 & $1999(+/-)^{*}$ \\
\hline Sex ratio (males/females) & $50.4 / 49.6$ & $52.5 / 47.5$ \\
Total sample & 1510 & 1524 \\
12-66 months & 1204 & $1204(1138 / 66)^{\star}$ \\
Nutrition sub-sample & 499 & 501 \\
12-66 months & 363 & $380(358 / 22)^{\star}$ \\
Biology sub-sample & 192 & 251 \\
$12-66$ months & 153 & $198(189 / 9)^{\star}$ \\
\hline
\end{tabular}

${ }^{*}$ Children aged 12-66 months having participated (+) or not participated $(-)$ in the national immunisation days.

months participated in NIDs and received vitamin A (Table 1).

\section{Xerophtbalmia}

No active corneal signs of xerophthalmia were identified in either 1997 or 1999 (we observed four eyes with leucoma in 1997 and three in 1999, not clearly attributable to vitamin A deficiency and not related to a measles history).

In 1997, 5.5\% (95\% CI 3.9-7.6) of children between 24 and 66 months of age were recognised as suffering from night blindness and 2.6\% (95\% CI 1.61-4.27) had Bitot spots. The global prevalence of clinical xerophthalmia, attested by night blindness and/or Bitot spots, was 6.9\% (95\% CI 5.1-9.2) (Table 2). This rate reached $10.5 \%$ for children aged between 36 and 47 months (Fig. 1).

The survey carried out in the same villages in March 1999 demonstrated a less dramatic situation relative to

Table 2 Variables associated with the groups of 12- to 66-month-old children studied in 1997 and 1999

\begin{tabular}{|c|c|c|c|c|}
\hline & 1997 & 1999 & $P$-value & OR $(95 \% \mathrm{Cl})$ \\
\hline \multicolumn{5}{|l|}{ Anthropometric status } \\
\hline $\mathrm{HAZ}<-2$ & $22.7 \%$ & $27.2 \%$ & 0.012 & \\
\hline WAZ $<-2$ & $24.0 \%$ & $25.1 \%$ & 0.53 & \\
\hline $\mathrm{WHZ}<-2$ & $12.8 \%$ & $11.5 \%$ & 0.32 & \\
\hline \multicolumn{5}{|l|}{ Vitamin A score } \\
\hline Animal sources (SD) & $0.65(1.70)$ & $0.75(1.26)$ & 0.38 & \\
\hline Vegetable sources (SD) & $19.46(17.11)$ & $15.37(11.81)$ & 0.0002 & \\
\hline \multicolumn{5}{|c|}{ Ophthalmologic examination } \\
\hline $\mathrm{XN}$ & $5.5 \%$ & $3.3 \%$ & 0.020 & $0.58(0.36-0.95)$ \\
\hline Bitot spots & $2.6 \%$ & $0.8 \%$ & 0.002 & $0.29(0.11-0.70)$ \\
\hline Xerophthalmia & $6.9 \%$ & $3.3 \%$ & $<0.0001$ & $0.46(0.32-0.73)$ \\
\hline \multicolumn{5}{|l|}{ MRDR test } \\
\hline Mean (SD) & $0.183(0.216)$ & $0.131(0.157)$ & 0.010 & \\
\hline Abnormal $(\geq 0.06)$ & $77.8 \%$ & $63.1 \%$ & 0.003 & $0.54(0.32-0.91)$ \\
\hline \multicolumn{5}{|l|}{ Retinol level } \\
\hline Mean $\left(\mu \mathrm{moll}^{-1}\right)(\mathrm{SD})$ & $0.38(0.18)$ & $0.42(0.22)$ & 0.037 & \\
\hline$<0.70 \mu \mathrm{moll}^{-1}$ & $94.8 \%$ & $89.4 \%$ & 0.069 & $0.38(0.13-1.08)$ \\
\hline$<0.35 \mu \mathrm{moll}^{-1}$ & $49.0 \%$ & $39.9 \%$ & 0.080 & $0.67(0.42-1.08)$ \\
\hline \multicolumn{5}{|l|}{ Infectious morbidity } \\
\hline Fever & $70.0 \%$ & $50.4 \%$ & $<0.0001$ & $0.44(0.32-0.60)$ \\
\hline Cough & $19.3 \%$ & $27.9 \%$ & 0.001 & $1.73(1.21-2.46)$ \\
\hline Diarrhoea & $38.8 \%$ & $23.6 \%$ & $<0.0001$ & $0.49(0.35-0.38)$ \\
\hline Measles & $10.2 \%$ & $11.3 \%$ & 0.64 & $0.99(0.61-1.62)$ \\
\hline
\end{tabular}

OR - odds ratio; Cl - confidence interval; HAZ - height-for-age Z-score; WAZ - weight-for-age Z-score; WHZ - weight-for-height Z-score; SD - standard deviation; XN - night blindness; MRDR - modified retinol dose response. 


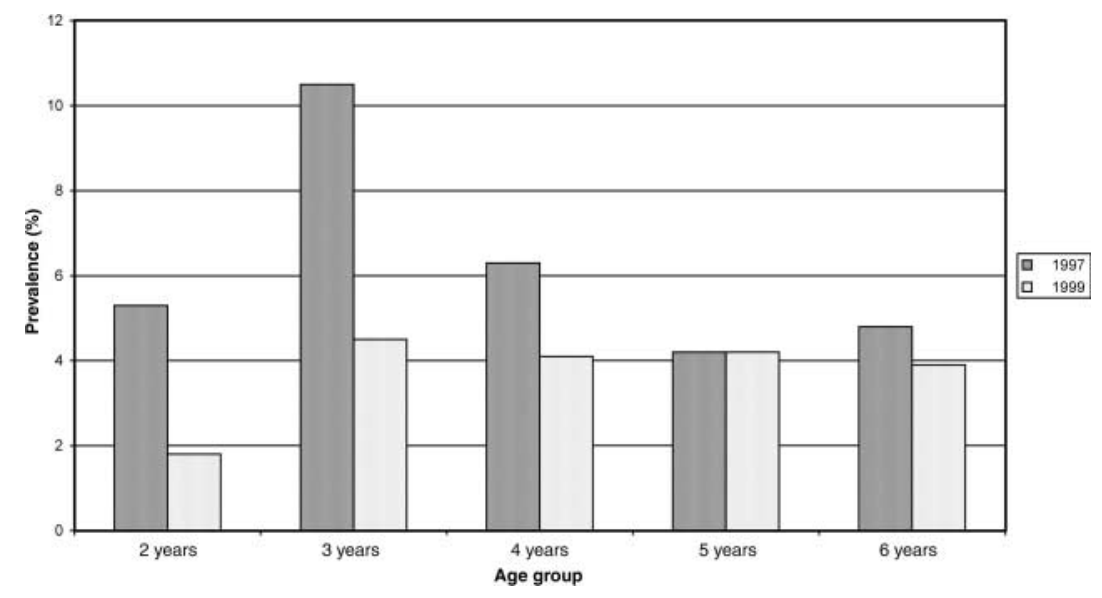

Fig. 1 Xerophthalmia prevalence per age group in 1997 and 1999

1997. The children were less likely to experience night blindness $(3.3 \%, 95 \%$ CI $2.1-5.2, P=0.02)$ or to have Bitot spots $(0.8 \%, 95 \%$ CI $0.3-2.0, P=0.002)$. Overall xerophthalmia prevalence was lower $(3.3 \%$, 95\% CI 2.1-5.2, $P=0.0001$ ) (Table 2) and reached $4.5 \%$ for children 36-47 months old (Fig. 1).

For children aged over 65 months, there were no significant differences between 1997 and 1999. Night blindness occurred in 3.2\% of this population in 1997 and $4.3 \%$ in $1999(P=0.59)$. The prevalence of Bitot spots was quite similar (2.1\%) between the two surveys in this age group. Xerophthalmia frequency was 5.3\% in 1997 and 4.8\% in 1999 ( $P=0.83)$ (Fig. 1$)$.

In 1999, children receiving the vitamin A supplementation showed a lower frequency of xerophthalmia than non-recipients in the same age group who had not received the nutrient $(3.0 \%, n=1138$ vs. $8.7 \%, n=66)$ (Table 3).

\section{Biological status}

In 1997, biological deficiency was far more frequent, since the MRDR test proved abnormal in $77.8 \%$ of children aged between 12 and 66 months (95\% CI 68.25-85.17). Some $94.8 \%$ of children had a serum retinol value of $<0.7 \mathrm{moll}^{-1}$ and $49.0 \%$ had a serum retinol value of $<0.35 \mu \mathrm{mol} \mathrm{l}^{-1}$. Mean MRDR was equal to 0.183 and mean serum retinol concentration was $0.38 \mu \mathrm{moll}^{-1}$ (Table 2).

Biological status also improved after vitamin A supplementation. In the second (1999) survey, 63.1\% (95\% CI 54.25-71.23) of children in the same age group had an abnormal response to the MRDR test, $89.4 \%$ had a serum retinol value of $<0.7 \mu \mathrm{moll}^{-1}$ and $39.9 \%$ a serum retinol value of $<0.35 \mu \mathrm{moll}^{-1}$. Mean MRDR ratio was equal to 0.131 and mean serum retinol concentration was $0.42 \mu \mathrm{moll}^{-1}$ (Table 2).

For children aged 65 months or above no significant differences were evidenced: between 1997 and 1999, the prevalence rates of abnormal MRDR test response were
76.0\% (95\% CI 49.0-92.0) and 65.0\% (95\% CI 35.6-86.9), respectively (Fig 1).

With regard to biological status, in the 1999 survey, when comparing children in the same age group (12 to 66 months) who had and not received vitamin A, we could not find any statistically significant difference because of the very small number of biological samples in the non-recipient group $(n=9)$ (Table 3 ).

\section{Antbropometric status}

The prevalences of critical values were not significantly different for weight insufficiency (WAZ $<-2$ for $24.0 \%$ of children in 1997 and $25.1 \%$ in 1999) and emaciation

Table 3 Variables associated with targeted, 12- to 66-month-old children receiving or not receiving vitamin A (1999 survey)

\begin{tabular}{|c|c|c|c|}
\hline & Recipients & Non-recipients & $P$-value \\
\hline \multicolumn{4}{|l|}{ Anthropometric status } \\
\hline $\mathrm{HAZ}<-2$ & $26.8 \%$ & $34.8 \%$ & 0.15 \\
\hline WAZ $<-2$ & $25.0 \%$ & $27.3 \%$ & 0.67 \\
\hline $\mathrm{WHZ}<-2$ & $11.5 \%$ & $12.1 \%$ & 0.88 \\
\hline \multicolumn{4}{|l|}{ Vitamin A score } \\
\hline Animal sources (SD) & $0.77(1.29)$ & $0.50(0.51)$ & 0.86 \\
\hline Vegetable sources (SD) & 15.25 & $17.87(14.16)$ & 0.33 \\
\hline \multicolumn{4}{|l|}{ Ophthalmologic examination } \\
\hline $\mathrm{XN}$ & $3.0 \%$ & $8.9 \%$ & 0.032 \\
\hline Bitot spots & $0.8 \%$ & $0.0 \%$ & \\
\hline Xerophthalmia & $3.0 \%$ & $8.7 \%$ & 0.037 \\
\hline \multicolumn{4}{|l|}{ MRDR test } \\
\hline Mean (SD) & $0.128(0.151)$ & $0.193(0.269)$ & 0.23 \\
\hline Abnormal $(\geq 0.06)$ & $59.6 \%$ & $60.0 \%$ & 0.76 \\
\hline \multicolumn{4}{|l|}{ Retinol level } \\
\hline Mean $\left(\mu \mathrm{moll}^{-1}\right)(\mathrm{SD})$ & $0.42(0.22)$ & $0.50(0.176)$ & 0.27 \\
\hline$<0.70 \mu \mathrm{moll}^{-1}$ & $90.4 \%$ & $80.0 \%$ & 0.60 \\
\hline$<0.35 \mu \mathrm{moll}^{-1}$ & $43.4 \%$ & $30.0 \%$ & 0.61 \\
\hline \multicolumn{4}{|l|}{ Infectious morbidity } \\
\hline Fever & $48.5 \%$ & $81.8 \%$ & 0.002 \\
\hline Cough & $27.9 \%$ & $63.6 \%$ & $<0.0001$ \\
\hline Diarrhoea & $21.7 \%$ & $54.5 \%$ & $<0.0001$ \\
\hline Measles & $11.7 \%$ & $2.3 \%$ & 0.49 \\
\hline
\end{tabular}

HAZ - height-for-age Z-score; WAZ - weight-for-age Z-score; WHZ weight-for-height $Z$-score; SD - standard deviation; XN - night blindness; MRDR - modified retinol dose response. 
(WHZ $<-2$ for $12.8 \%$ of children in 1997 and $11.5 \%$ in 1999) between the two surveys. However, we observed a significant worsening of the chronic malnutrition indicator in the 1999 survey (HAZ $<-2$ for $22.7 \%$ of children in 1997 and 27.2\% in 1999) (Table 2).

\section{Food survey}

In 1997, for children aged from 12 to 66 months, the animal and vegetable vitamin A scores were 0.65 and 19.46, respectively. There was no significant change in 1999 for the animal vitamin A score (0.75 occasions, $P=0.38$ ), but the vegetable sources of beta-carotene were less frequent (15.37 occasions, $P<0.001$ ) (Table 2 ).

\section{Infectious morbidity}

When comparing children (aged 12 to 66 months) in the 1997 and 1999 populations, children in the 1999 survey had experienced fewer fever episodes during the preceding two weeks (50.4\% vs. 70.0\%, $P<0.0001)$ and less diarrhoea (23.6\% vs. 38.8\%, $P<0.0001$ ). They had experienced a little more coughing (27.9\% vs. $19.8 \%$, $P=0.001)$ and a comparable frequency of measles episodes (11.3\% vs. 10.2\%) (Table 2).

Moreover, in 1999, comparison among children of the same age group (12 to 66 months) who received and did not receive vitamin A revealed that recipients had experienced fewer fever episodes during the preceding two weeks ( $48.5 \%$ vs. $81.8 \%, P<0.01$ ), less coughing (27.9\% vs. 63.6\%, $P<0.001)$ and fewer diarrhoea events (21.7\% vs. $54.5 \%, P<0.001$ ) (Table 3 ).

\section{Comments and discussion}

These two surveys suggest that the clinical and biological vitamin A status of children aged between 12 and 66 months who were targeted by NIDs improved between 1997 and 1999. Mass distribution of vitamin A appears to reduce clinical xerophthalmia and to be associated with a reduction in the frequency of other related illnesses.

Children aged 66-84 months should not be compared with younger individuals since epidemiological studies suggest that the risk of vitamin A deficiency, especially for xerophthalmia, is very different in the two age groups ${ }^{14}$. Nevertheless, it was possible to compare children aged 66-84 months in the 1997 and 1999 surveys: no change could be found, either clinical or biological.

\section{Xeropbthalmia}

Night blindness is a subjective but extremely valuable indicator for evaluating vitamin A deficiency. Nevertheless, the diagnosis of night blindness rests on questions to the children's mothers and the figures should therefore be interpreted with caution. It is important to note that, in all of the villages investigated, local terms existed for the condition, indicating a good knowledge of the phenomenon.
Even if the prevalence of night blindness decreased considerably in 1999, the rate of $3.3 \%$ is still higher than the threshold of $1 \%$ established by the WHO as defining a public health problem in a given community.

Bitot spot is an early pathognomonic clinical sign of vitamin A deficiency. This sign has to be interpreted cautiously because it does not necessarily indicate a current vitamin A deficiency.

\section{Biochemical vitamin A deficiency}

This deficiency is found far more frequently than clinical xerophthalmia, which corresponds to a relatively late and severe stage: serum retinol value only reflects the vitamin A reserves when these have largely been depleted. Level of retinol $<0.7 \mu \mathrm{moll}^{-1}$ is classically classified as weak deficiency, whereas a deficiency is said to be severe when the retinol level falls below $0.35 \mu \mathrm{moll}^{-1}$. It is a very sensitive but not specific biological test. A deficit can be aggravated by insufficient protein supply and infection, which affect retinol mobilisation. The DR to retinol ratio reflects the hepatic stock of vitamin A and can be considered as a more reliable indicator than retinol itself. This ratio improved between the two surveys but is still much higher than the alarm threshold defined by the WHO, which is $20 \%$ of children ${ }^{13}$.

The prevalence of serum retinol $<0.70 \mu \mathrm{moll}^{-1}$ was astonishingly high at baseline (94.8\%) and continued to be high after vitamin A supplementation. Mali is one of the countries with the highest known prevalence of subclinical vitamin A deficiency among children. Nevertheless, a similar presentation (93\% were deficient at the $0.70 \mu \mathrm{moll}^{-1}$ threshold and $19.7 \%$ at the $0.35 \mu \mathrm{moll}^{-1}$ threshold) has been observed in Kivu Province in Zaire ${ }^{15}$ among pre-school children who presented a prevalence of night blindness of $0.7 \%$, a lower rate than found in Bandiagara Circle.

The high level of abnormal results for the MRDR test may also be compared with those measured in other African countries. In 1996, a study carried out in Zambia by Kafwembe et al. on 104 children found a proportion of $78 \%$ producing abnormal results in the MRDR test ${ }^{16}$.

Measurement of changes in vitamin A status following a vitamin A intervention has become an important issue when looking at the performance of relevant and sensitive indicators. Suitable determinants to consider when designing an intervention study, both for the population being followed and in answering the query posed, may include the following: estimated liver weight, amount of vitamin A administered, estimated spontaneous loss and study duration. Several vitamin A intervention trials have been evaluated to provide relevant models or markers to predict changes in the average individual enrolled in a study. The MRDR test has often been shown to be a legitimate method of measuring nutrient status increment with large doses of vitamin A 
used in the intervention, even with a late post-intervention time point.

Considering the above criteria, the total amount of vitamin A administered, the ascertained absorption and utilisation rates, the storage efficiency of a large dose $(\sim 40 \%)^{17}$ and the time interval between data collection ${ }^{18}$, a model has been applied using data from studies in children ${ }^{18,19}$. For example, using the study by Tanumihardjo et $a l .{ }^{18}$, who administered a single dose of 200000 IU of vitamin A to children weighing $\sim 12.5 \mathrm{~kg}$, the following was calculated using a $75 \%$ effective delivery and an estimated $40 \%$ of large dose stored. Considering a recommended intake of $400 \mu \mathrm{g} \mathrm{day}^{-1}$ for children $4-8$ years old, which overestimates average needs, an MRDR test performed 21-28 days after the dose would still detect appreciable reserves.

In the surveys carried out in Bandiagara Circle, the MRDR test performed 4-5 months after supplementation showed fewer abnormalities than in a similar population two years before. It is difficult to apply the Tanumihardjo model as the individuals were not the same in both surveys. A cohort study establishing vitamin A stocks every two months for six months after supplementation and considering dietary intakes and infectious events that may reduce serum retinol concentration could better test the adequacy of this model.

\section{Antbropometric status}

We did not observe more appropriate anthropometric indicators in 1999 that could explain the improvement in vitamin A status. Indeed, WHZ and WAZ scores were quite similar and HAZ scores were significantly worse than in 1997. This last score, reflecting chronic malnutrition, is generally a consequence of inadequate food and/or illnesses lasting for a relatively long period. The situation is permanently bad and figures from the two surveys are close to those noted in the demographic and health study $^{20}$ of children below 3 years old, which recorded 27.6\% of stunted children in Mopti region.

In the given time frame, we acknowledge that no significant difference in weight-for-age and weight-for-height distribution would be expected, as no specific determinant act of nature or man had occurred during the year.

\section{Nutrition}

Food availability can vary depending on season and year, and this could influence children's nutritional status and may represent an important potential bias that has to be discussed. Both studies were carried out in the dry season, when there is a shortage of sources of vitamins, as few fresh vegetable foods are available. Nevertheless, consumption rates were not significantly different between 1997 and 1999 for animal foods rich in vitamin A and were inferior for vegetable foods rich in vitamin A, suggesting that the average intake of foods rich in this vitamin did not improve.

\section{Morbidity events}

Associated illnesses appear more frequently in the two studies than found in the demographic and health evaluation for Mopti region ${ }^{20}$. This last evaluation, performed on children below 3 years of age, found prevalences of $35.2 \%$ for fever episodes, $11.8 \%$ for coughing and $29 \%$ for diarrhoea. The timing of the vitamin A studies may have played a role, since, in the period of March to April, dryness and the Harmattan wind provide favourable conditions for respiratory and infectious conditions to emerge.

\section{Non-recipients in 1999}

In 1999, comparing non-recipients with recipients in the 12- to 66-month age group, we observed lower vitamin A status and many more morbidity events in the nonrecipient group. These results need to be interpreted with extreme caution and care because non-recipients could be otherwise disadvantaged. Non-recipients could be exposed to a more critical nutritional status and therefore be at a much higher risk of vitamin A deficiency than the children covered in the programme. These children were recognised as having slightly less favourable anthropometric indicators but with no significant difference in vitamin A intake (Table 3).

These results argue that the inclusion of vitamin A supplementation into immunisation campaigns or programmes and other similar vehicles should be a suitable and efficient opportunity for those areas where vitamin A deficiency remains an important public health problem or emergency.

\section{Acknowledgements}

This study was financed by the German 'Primary Health Care' technical co-operation project of the GTZ (Deutsche Gesellshaft für Technische Zusammenarbeit) in Sévaré. We thank Dr V. Joret, team leader of GTZ, for his help during the two surveys.

We would like to thank the Mopti Regional Health Directorate and the team at the Centre de Santé du Cercle of Bandiagara Circle for their assistance. We also thank Dr H. Faure, Biochemistry Laboratory, University of Grenoble for analytical assistance.

\section{References}

1 Goodman T, Dalmiya N, de Benoit B, Schultink W. Polio as a platform: using national immunization days to deliver vitamin A supplements. Bull. World Health Org. 2000; 78: 305-14.

2 West KP Jr. Extent of vitamin A deficiency among preschool children and women of reproductive age. J. Nutr. 2002; 132: 2857S-66S.

3 Beaton GH, Martorell R, Aronso KJ. Vitamin A supplementation and morbidity and mortality in developing countries. Food Nutr. Bull. 1994; 15: 282-9.

4 Humphrey JH, West KP, Sommer A. Vitamin A deficiency 
and attributable mortality among under-5-year-old. Bull. World Health Org. 1992; 70: 228-32.

5 United Nations Children's Fund (UNICEF). Progress of Nations. New York: UNICEF, 1999.

6 Traoré L, Banou A, Sacko D, Malvy D, Schémann JF. Les stratégies de lutte contre les déficits en vitamine A. Santé 1998; 8: 158-62.

7 World Health Organization (WHO). Methods of Assessment of Avoidable Blindness. WHO Offset Publication No. 54. Geneva: WHO, 1980

8 Direction Nationale de la Statistique et de l'Information (DNSI). Recensement général de la population et de l'habitat du Mali (RGPH). Bamako, Mali: DNSI, 1987.

9 WHO Working Group. Use and interpretation of anthropometric indicators of nutritional status. Bull. World Health Org. 1986; 64: 929-41.

10 Helen Keller International (HKI). How to Use the HKI Frequency Method to Assess Community Risk of Vitamin A Deficiency. New York: HKI, 1994.

11 Tanumihardjo SA, Muhilal ER, Yuniar Y, Permaesih D, Sulaiman Z, Karyadi D, et al. Vitamin A status in preschool age Indonesian children as assessed by the MRDR assay. Am. J. Clin. Nutr. 1994; 60: 142-7.

12 Tanumihardjo SA, Koelner PG, Olson JA. The modified relative dose response assay as an indicator of vitamin $\mathrm{A}$ status in a population of well-nourished American children. Am.J. Clin. Nutr. 1994; 60: 148-52.

13 World Health Organization (WHO). Indicators for Assessing Vitamin A Deficiency and Their Application in Monitoring and Evaluating Intervention Programmes. Micronutrient Series. Geneva: WHO, 1996.

14 Sommer A. Vitamin A Deficiency and Its Consequences, A Field Guide to Detection and Control, 3rd ed. Geneva: World Health Organization, 1995.

15 Donnen P, Brasseur D, Dramaix M, Vertongen F, Ngoy B, Zihindula $\mathrm{M}$, et al. Vitamin A deficiency and protein-energy malnutrition in a sample of preschool age children in the Kivu Province in Zaïre. Eur. J. Clin. Nutr. 1996; 50: 456-66.

16 Kafwembe EM, Sakwa TY, Manyando O, Mwandu D, Chipipa J, Chipaila P. The vitamin A status of Zambian children attending an under five clinic as evaluated by the Modified Relative Dose Response (MRDR) test. Int. J. Vit Nutr. Res. 1996; 66: 190-6.

17 Haskell MJ, Handelman GJ, Peerson JM, Jones AD, Rabbi MA, Awal MA, et al. Assessment of vitamin A status by the deuterated-retinol-dilution technique and comparison with hepatic vitamin A concentration in Bangladeshi surgical patients. Am. J. Clin. Nutr. 1997; 66: 67-74.

18 Tanumihardjo SA, Permaesih D, Muherdiyantinindsih, Rustan E, Rusmil K, Fatah AC, et al. Vitamin A status of Indonesian children infected with Ascaris lumbricoides after dosing with vitamin A supplements and albendazole. J. Nutr 1996; 126: 451-7.

19 Tanumihardjo SA. Can lack of improvement in vitamin A status indicators be explained by little or no overall change in vitamin A status of humans? J. Nutr. 2001; 131: 3316-8.

20 Ministère de la Santé. EDS: Enquête Démographique et de Santé. Bamako, Mali: Ministère de la Santé, 1996. 